
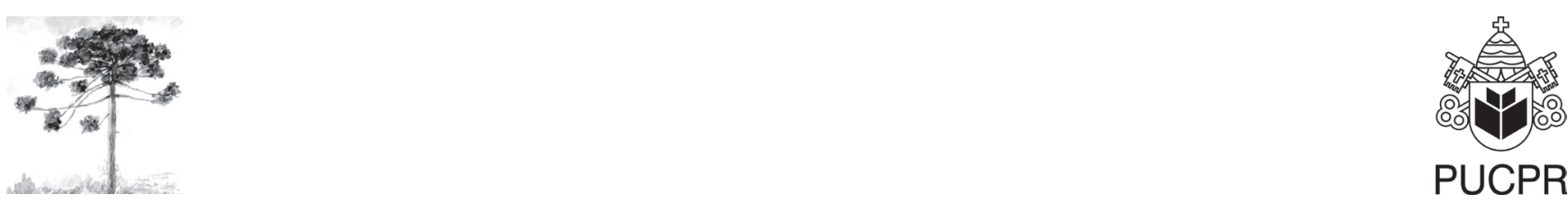

\title{
AVIFAUNA, MASTOFAUNA E REPTILIA DO PARQUE ESTADUAL MASSAIRO OKAMURA, CUIABÁ, MT
}

\author{
Avifauna, mammalian and reptile State Park Massairo Okamura, Cuiabá, MT
}

\author{
Jorge Luiz de Arruda
}

Licenciado e Bacharel em Ciências Biológicas pela Universidade de Cuiabá (UNIC), Especialista em Gestão Ambiental pela Universidade Gama Filho, servidor público estadual lotado na Secretaria de Estado de Saúde de Mato Grosso, Cuiabá, MT - Brasil, e-mail: jorgeluizdearruda@ig.com.br

\section{Resumo}

O Parque Estadual Massairo Okamura, criado pela Lei n. 7.506/01, contempla em seu interior várias espécies de seres vivos, desde os invertebrados até os vertebrados. Entre os vertebrados, podemos destacar os mamíferos e os pássaros, objetos de nosso estudo. Com a presença de pessoas nesse local, pode-se constatar que muitos animais procuram não se fazer presente durante o dia, sendo observados nos crepúsculos matutino e vespertino, ou seja, em um horário em que a presença humana quase não se faz presente. A falta de corredores artificiais para os animais se deslocarem dentro e fora do parque é com toda certeza o maior obstáculo para a sua sobrevivência, sendo as espécies de primatas como o sagui e o gambá, que é um marsupial, as principais vítimas de acidentes e mortes causadas pela não construção desses corredores. Desenvolver uma estrutura ambiental apta a receber esses seres é um desafio a ser revisto pelas autoridades constituintes e de certa maneira envolve a comunidade que ali se faz presente. Verificamos a presença de pessoas de outras regiões de Cuiabá, como, por exemplo, o Centro, e até mesmo oriundas do município vizinho, Várzea Grande. Para a conservação e manutenção do parque, os setores públicos envolvidos e a sociedade civil organizada devem trabalhar em conjunto para a preservação de um ecossistema tão importante inserido em uma área limitada, como é o caso do Parque Massairo Okamura.

Palavras-chave: Conservação. Corredores. Mamíferos.

\section{Abstract}

The State Park Massairo Okamura created by Law n. 7.506/01 includes inside various living species from invertebrates to vertebrates. Among the vertebrates we can highlight the mammals and birds, the object of our study. With the presence of people on this site can be seen that many animals try 
not to be present during the day, being observed in the morning and evening twilight, or in a time when human presence is hardly present. The lack of artificial corridors for animals to move in and out of the park is almost certainly the greatest obstacle to their survival, and species of primates such as marmosets and opossum is a marsupial, the main victims of accidents and deaths not caused by construction of these corridors. Develop an environmental structure able to receive these beings is a challenge to be reviewed by the constituents and somehow involving the community that there is present. Verified the presence of people from other regions of Cuiaba, for example, the Downtown and even coming from the neighboring district, Várzea Grande. For the preservation and maintenance of the park public sectors involved with the organized civil society must work together to preserve an important ecosystem as inserted in a limited area such as Park Massairo Okamura.

Keywords: Conservation. Corridors. Mammals.

\section{INTRODUÇÃO}

Desde o advento da criação do Parque Estadual Massairo Okamura, em 2001, pouca coisa se sabe a respeito desse ambiente, portanto, com este levantamento preliminar, a direção do Parque e a Secretaria de Estado de Meio Ambiente (Sema) poderão definir ações de impacto para a proteção desse bioma. Sabendo que, a partir do momento em que limitam uma área para obter o controle das espécies ou até mesmo a sua preservação, alguns cuidados devem ser tomados para que essa área consiga reproduzir condições ambientais para os organismos vivos existentes.

Entre esses cuidados está: a criação de lei de controle que viabilizará a permanência dos seres vivos em um determinado espaço; criação de corredores ecológicos artificiais para a locomoção desses seres no espaço delimitado; visitação de pessoas no recinto, tomando cuidado para que não venham a danificar e nem exterminar nenhuma espécie que viva no local.

Um plano de manejo deve ser desencadeado a partir da transformação dessa unidade em um parque ou uma reserva natural, sendo feita a identificação por meio de uma contagem populacional dos organismos pertencentes àquela área antes e depois da transformação em parque ou reserva natural.

A verificação de agentes biológicos atuando no recinto é um fator predominante em virtude de que, em alguns casos, o desaparecimento de determinadas espécies se acentua drasticamente em razão do afastamento ou extinção de outras espécies que fazem parte da cadeia alimentar dos organismos ali presentes.

Pesquisar mamíferos e pássaros dentro de uma unidade de conservação tem sido um trabalho laborioso, em virtude de que, em muitos casos, a visualização de certas espécies não nos dará a certeza de qual espécie realmente estamos visualizando. Utilizar as técnicas de identificação, como a imagem fotográfica, e até mesmo a identificação a partir de livros especializados, confrontando com opiniões de especialistas das áreas, será um marco para o procedimento deste trabalho.

Existem atualmente 4.809 espécies de mamíferos descritos em todo o mundo. O Brasil detém 524 espécies, aproximadamente 11\% do total de espécies descritas (1). A biodiversidade que o Brasil possui é pouco conhecida e uma parte dela encontra-se ameaçada por atividades humanas, portanto, é urgente a ampliação do conhecimento desse rico patrimônio nacional (2). Geralmente, os pequenos roedores e marsupiais são os grupos mais estudados (3).

A destruição acelerada do meio ambiente compromete a existência de várias espécies brasileiras. A ação antrópica leva a uma diminuição e fragmentação das áreas naturais, obrigando a fauna silvestre a viver em áreas cada vez menores (4).

Alguns métodos são bastante utilizados em levantamentos de mamíferos de médio e grande porte, um deles é o método de registro de pegadas (rastros). O registro de pegadas pode ser obtido por meio da procura aleatória pela área de estudo ou por meio de parcelas de areia que servem como armadilhas para se obter o registro. Em procuras aleatórias por rastros, um fator a ser considerado é a idade do rastro, pois os mais antigos são mais difíceis de identificar (5).

Outro método utilizado é o uso de armadilhas fotográficas automáticas. Esse recurso fotográfico é recente e vem sendo utilizado para 
responder questões ecológicas, em estimativas de tamanho populacional, no estudo de habitats, período de atividades, entre outros (6).

A entrevista também é um método muito utilizado para complementar os dados de campo. Apesar de ser um método duvidoso, pode ser proveitoso quando aplicado a algumas pessoas, como, por exemplo, caçadores e moradores do local que tenham um conhecimento da fauna (5).

O estudo da avifauna é de vital importância para a manutenção e preservação ambiental. A observação, o monitoramento e a compreensão de seus hábitos alimentares, reprodutivos, etc., serve-nos como um importante bioindicador (7).

Atualmente, estão descritas mais de 9.000 espécies de aves, divididas em 27 ordens atuais (8). No Brasil, o número de espécies conhecidas é de aproximadamente 1.677 (9), podendo ser separadas em grupos funcionais ou em guildas, predadoras, piscívoras, insetívoras, nectívoras, frugívoras, granívoras, carnívoras e onívoras.

Este trabalho foi desenvolvido no sentido de se conhecer as espécies de animais (aves, mamíferos e répteis) encontradas dentro e no entorno do Parque Estadual Massairo Okamura, por isso a necessidade de se fazer uma pesquisa detalhada das espécies, determinando a ordem taxonômica destas conforme a literatura científica atualizada.

Além disso, conhecendo as espécies que habitam o parque estadual e o seu entorno, os órgãos competentes poderão definir políticas de conservação e preservação do ambiente a partir de um plano de manejo, criação de corredores ecológicos e outras alternativas que facilitarão a sobrevivência das espécies avistadas ou não nos limites do parque.

\section{MATERIAIS E MÉTODOS}

\section{Metodologia}

Este trabalho foi desenvolvido por meio de visitas no local procurando identificar primeiramente as espécies mais conhecidas, sendo criada uma tabela classificando os seres vivos conforme a taxonomia científica (ordem, classe, família e espécie).

As visitas ao parque foram realizadas nos fins de semana e feriados, de janeiro de 2005 até outubro de 2007 e de janeiro de 2008 a dezembro de 2009. Nessas visitas, obtivemos informações de algumas espécies por intermédio dos funcionários que ali trabalhavam, dando referência de onde esses animais poderiam ser localizados e avistados. Tais visitas totalizaram 400 horas de observações.

Foram utilizados máquina fotográfica e bloco de anotações. Para a identificação das espécies, foram realizadas consultas bibliográficas em revistas especializadas e também um estágio no Zoológico da Universidade Federal de Mato Grosso (UFMT), o que facilitou a identificação das espécies dentro do parque estadual.

\section{Área de estudo}

O trabalho foi desenvolvido na área do Parque Estadual Massairo Okamura e no seu entorno. A entrada principal do parque fica na Avenida Historiador Rubens de Mendonça, s/n (Avenida do CPA), bairro Morada do Ouro e sua parte posterior fica na Avenida Leônidas P. Mendes (Figura 1), estando localizado na região norte de Cuiabá, na Grande Morada da Serra.

Com a elaboração da Lei Estadual n. 7.313/00 (10), criou-se novamente a reserva ecológica; e com a Lei n. 7.506/01 (11) transformou-se a reserva ecológica em um parque estadual. A partir de então, o governo do estado assumiu o compromisso da gestão da área, promovendo a construção de cercas no perímetro da unidade de conservação, como também, posteriormente, alocando recursos próprios, necessários para transformar parte da sua área (11 hectares) em um local de lazer e recreação para a população, nos moldes do Parque Mãe Bonifácia (12).

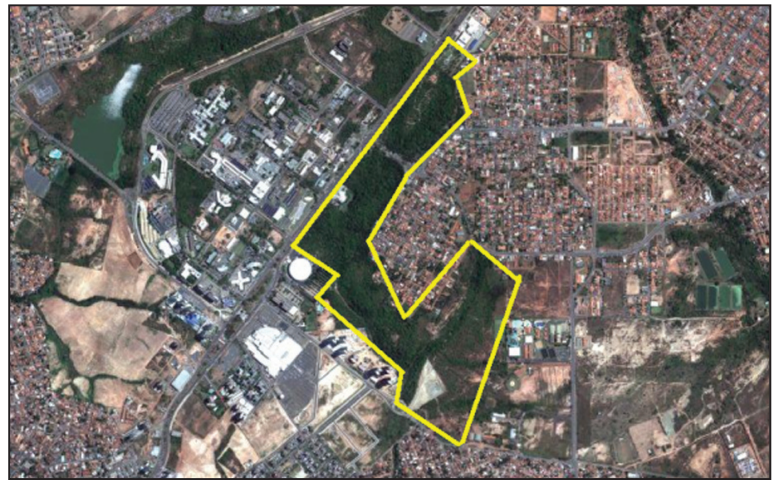

FIGURA 1 - Vista aérea do Parque Estadual Massairo Okamura

Fonte: Prefeitura Municipal de Cuiabá, 2007 (13). 


\section{RESULTADOS E DISCUSSÕES}

Como o Parque Estadual Massairo Okamura está localizado em uma região de recursos naturais e que oferece grandes possibilidades de desenvolvimento econômico regional e nacional, ele apresenta também uma parcela muito variável de áreas verdes tanto dentro como no entorno e essa parcela é, sem dúvida alguma, fator determinante na diversidade da fauna e da flora presentes na região.

O parque tem flora e fauna exuberantes e, com isso, oferece recursos alimentares, locais para nidificação e refúgios disponíveis, onde as aves e os mamíferos possam se estabelecer.

Podemos definir a área trabalhada com quatro tipos de vegetações bem definidas, e na parte superior, onde fica a sua entrada, podemos classificar a vegetação do tipo Cerrado pela presença de árvores típicas, como lixeira (Curatella americana), cumbaru (Dipteryx alata), chico-magro (Guazuma ulmifolia) e outras.

Ao centro, com a presença das nascentes, encontramos a vegetação Ciliar. Destacamos a sua presença, principalmente, em áreas com muita água, ou seja, perto de nascentes, córregos e rios. O terceiro tipo de vegetação encontrada é do tipo transição, ou seja, uma mistura de Cerrado com Mata Ciliar, sendo encontrado nessa área o angicovermelho (Parapiptadenia rigida (Benth.) Brenan), que se apresenta em grandes proporções.

A quarta vegetação, e que traz preocupação, é a exótica, sendo duas espécies presentes: a leucena (Leucaena spp.), que é uma planta utilizada para alimentação do gado e se apresenta em vargens onde se dispersam as suas sementes. A leucena está presente em grande extensão do parque, sem nenhum tipo de manejo, e, com isso, poderá com o tempo eliminar as espécies nativas, a partir do efeito de borda que vem realizando, por exemplo, diminuindo a incidência de raios solares no interior do parque.

A outra espécie exótica é a teca (Tectona grandis), que é originária da Ásia, tem folhas grandes e sua reprodução é feita por meio dos seus frutos. Tais frutos são do tipo drupa e em suas cavidades estão as sementes que são dispersas no ar e, auxiliadas pelo vento, alcançam grandes distâncias (13).

O parque possui duas nascentes que, no período de estiagem, ficam secas, causando assim falta de água em determinado período do ano. Poderíamos dizer que os animais se encontram servidos de pelo menos um item básico para a sua sobrevivência: a água, porém, muitos animais procuram deslocar-se para outras áreas localizadas nas proximidades, nesse ponto temos duas convergências que eles fazem.

A primeira se encontra na frente do parque estadual, tendo como ponto de separação a Avenida Historiador Rubens de Mendonça (Av. do CPA), onde se encontra a $13^{\text {a }}$ Brigada de Infantaria Motorizada e a Loja Maçônica. Nesse ponto, temos uma área de Mata Ciliar com presença de outra nascente que desemboca também no Córrego do Barbado. A primeira vista podemos destacar que essa área deve conter muitos atrativos para os seres vivos, principalmente, alimentação. Contudo, para que esses seres atravessem a área limite, deverão passar pela avenida movimentada até chegar a outro ponto desejado. Com isso percebemos alguns animais mortos nesse ponto.

Outro ponto de estrangulamento para esses seres vivos é a Avenida Professor Djalma Ferreira, que serve de entrada para o bairro Morada do Ouro. Quando os animais do parque procuram atravessar esse ponto a dificuldade é imensa, registrando-se também casos de óbito.

O que mais atrai os animais a esse ponto é, sem dúvida, a grande quantidade de água acumulada em um ponto onde as três nascentes se juntam. Somase a isso, também, a grande possibilidade de obtenção de alimentos nessa área, graças, principalmente, ao não acesso de pessoas ao local por conta de intensa mata, do terreno acidentado e do um declínio bem acentuado.

Verifica-se a travessia, no período noturno, de animais como os saguis (Callithris sp.), gambás (Didelphis marsupialis) e até mesmo as lebrinhas (tapiti). Os saguis utilizam as árvores como ponto de locomoção, mas quando chegam ao limite do parque o acesso a elas fica um tanto dificultoso, existindo uma distância considerada entre as árvores do parque e as dos canteiros.

\section{Espécies localizadas no Parque Estadual Massairo Okamura}

\section{Classe Ave}

A pesquisa identificou 11 gêneros de aves, 16 famílias, com 27 espécies mais comuns, no parque estadual e no seu entorno. Pode-se constatar dentro do parque uma grande proliferação de aves, animais vertebrados e outros (Tabela 1). 
TABELA 1 - Relação de espécies de aves avistadas no perímetro do parque

\begin{tabular}{|c|c|}
\hline \multicolumn{2}{|c|}{ Aves } \\
\hline Ordem/família/nome científico & Nome vulgar \\
\hline \multicolumn{2}{|c|}{ Anseriformes } \\
\hline \multicolumn{2}{|l|}{ Anatidae } \\
\hline Traminophilus doliatus & choca-do-pantanal \\
\hline \multicolumn{2}{|c|}{ Apodiformes } \\
\hline \multicolumn{2}{|l|}{ Trochilidae } \\
\hline Antracotorax nigricolis & beija-flor \\
\hline Clorostilbon aureoventris & beija-flor-do-peito-branco \\
\hline Phaetornis petrei & beija-flor-tesourinha \\
\hline \multicolumn{2}{|c|}{ Columbiformes } \\
\hline \multicolumn{2}{|l|}{ Columbidae } \\
\hline \multicolumn{2}{|l|}{ Leptotila verrauxi } \\
\hline \multicolumn{2}{|l|}{ Columbia livia } \\
\hline \multirow{2}{*}{\multicolumn{2}{|c|}{$\begin{array}{l}\text { rolinha-roxa ou rolinha-caldo-de-feijão } \\
\text { rolinha fogo-apagou }\end{array}$}} \\
\hline & \\
\hline \multicolumn{2}{|c|}{ Cuculiformes } \\
\hline \multicolumn{2}{|l|}{ Cuculidae } \\
\hline \multicolumn{2}{|l|}{ Crotophaga ani } \\
\hline \multirow{2}{*}{\multicolumn{2}{|c|}{$\begin{array}{l}\text { anu-branco } \\
\text { alma-de-gato }\end{array}$}} \\
\hline & alma-de-gato \\
\hline \multicolumn{2}{|c|}{ Falconiformes } \\
\hline \multicolumn{2}{|l|}{ Falconidae } \\
\hline \multirow{2}{*}{\multicolumn{2}{|c|}{ Micrastur ruficollis }} \\
\hline & \\
\hline \multicolumn{2}{|l|}{ Rollidae } \\
\hline \multirow{2}{*}{\multicolumn{2}{|c|}{ Rallus maculatus }} \\
\hline & \\
\hline \multicolumn{2}{|l|}{ Tyrannidae } \\
\hline \multicolumn{2}{|l|}{$\begin{array}{l}\text { Pitangus sulfuratus } \\
\text { Fringillidae }\end{array}$} \\
\hline Oryzoborus angolensis & curió \\
\hline Passer domesticus & pardal \\
\hline Furnaridae & \\
\hline $\begin{array}{l}\text { Furnarius rufus } \\
\text { Troglodytidae }\end{array}$ & joão-de-barro \\
\hline $\begin{array}{l}\text { Turdus rufiventris } \\
\text { Thraupidae }\end{array}$ & sabiá-laranjeira \\
\hline Traupir palmarum & sanhaço \\
\hline Volatinia jacarina & tiziu \\
\hline & \\
\hline Picidae & \\
\hline Picumnus sp. & pica-pau-anão \\
\hline Veniliornis passerinus & picapauzinho \\
\hline & \\
\hline Podicipedidae & \\
\hline Pyrocephalus rubinus & príncipe \\
\hline & \\
\hline Psittacidae & \\
\hline Amazona aestiva aestiva & papagaio-verdadeiro \\
\hline Pyrrbura spp. & periquito \\
\hline & \\
\hline Strigidae & \\
\hline Otus choliba & corujinha-do-mato \\
\hline Nyctibiidae & \\
\hline Nictibius sp. & urutau \\
\hline
\end{tabular}




\section{Classe Mammalia}

Foram identificados seis gêneros, sete famílias, com sete espécies de mamíferos avistados dentro do parque (Tabela 2).

\section{Classe Reptilia}

Além dos mamíferos e aves supracitados, destacamos, a título de conhecimento, que dentro do parque estadual encontra-se um réptil, da ordem dos crocodilianos, que fica próximo de uma das nascentes: o jacaré-do-pantanal. No parque há também outras espécies, conforme a Tabela 3.
Foram localizados, também, insetos como: abelhas, besouros, borboletas, cigarras, formigas, lagartas, cupins, lesmas, centopeias, piolhos-decobra, vespas, tatuzinhos de jardim, aranhas, etc. Caberá ser realizado um trabalho de pesquisa na posteridade sobre essas espécies, definindo a sua taxonomia completa.

Identificamos algumas espécies vegetais localizadas dentro do parque, essas espécies são de características diversas, predominando as do Cerrado, entre as quais podemos identificar a lixeira (Curatella americana); o cumbarú (Dipteryx alata); o angicovermelho (Parapiptadenia rigida (Benth.) Brenan), que se apresenta em grande quantidade dentro do parque; o ipê-amarelo (Tabebuia serratifolia); o ipê-roxo (Tabebuia avellanedae); e o ipê-branco (Tabebuia roseo-alba).

TABELA 2 - Relação de mamíferos avistados no parque estadual

\begin{tabular}{|c|c|}
\hline \multicolumn{2}{|l|}{ Mammalia } \\
\hline Ordem/família/nome científico & Nome vulgar \\
\hline \multicolumn{2}{|l|}{ Carnívora } \\
\hline \multicolumn{2}{|l|}{ Procyonidae } \\
\hline Procyon cancrivorus & mão-pelada \\
\hline \multicolumn{2}{|l|}{ Edentata } \\
\hline \multicolumn{2}{|l|}{ Dasipodidae } \\
\hline Euphractus spp. & tatupeba \\
\hline \multicolumn{2}{|l|}{ Logomorpho } \\
\hline \multicolumn{2}{|l|}{ Leporidae } \\
\hline Sylvilagus brasiliensis & lebre ou tapiti \\
\hline \multicolumn{2}{|l|}{ Marsupialia } \\
\hline \multicolumn{2}{|l|}{ Didelphidae } \\
\hline Didelphis marsupialis & gambá \\
\hline \multicolumn{2}{|l|}{ Rodentia } \\
\hline \multicolumn{2}{|l|}{ Hydrochaeridae } \\
\hline Dasyproctidae & capivara \\
\hline Dasyprocta sp. & cotia ou cutia \\
\hline Primates & \\
\hline \multicolumn{2}{|l|}{ Callithricidae } \\
\hline Callithris argentata & sagui \\
\hline
\end{tabular}

TABELA 3 - Relação de répteis identificados no parque estadual

\begin{tabular}{lcc}
\hline \multicolumn{2}{c}{ Reptilia } & \\
\hline Alligatoridae & Crocodylia & \\
\hline Caiman crocodilus yacare & & jacaré \\
\hline Iguanidae & Squamata & iguana, sinimbu \\
\hline $\begin{array}{l}\text { Iguana iguana } \\
\text { Teiidae }\end{array}$ & teiú \\
$\begin{array}{ll}\text { Tupinambis sp. } \\
\text { Tropiduridae }\end{array}$ & calango \\
\hline $\begin{array}{l}\text { Tropidurus itambere } \\
\text { Gekkonidae }\end{array}$ & lagartixa \\
\hline
\end{tabular}




\section{CONSIDERAÇÕES FINAIS}

Outras pesquisas devem se realizadas para alcançar outras espécies de animais que se encontram no ambiente e, além das espécies verificadas preliminarmente, outras devem viver dentro do local, sendo muitas destas com hábitos noturnos e esta pesquisa foi realizada no período diurno (manhã e tarde).

Com a variação da flora existente e a localização de nascentes no parque estadual, com toda a certeza deve haver espécies de animais de pequeno e médio porte vivendo escondidos ou utilizando o parque no período noturno, para se locomover e se alimentar, pois no período diurno a presença humana deve incomodar muito esses seres vivos.

A partir deste estudo, in loco, pode-se definir que, com a criação do Parque Ecológico Massairo Okamura, a fauna desselocal passou a teruma sobrevida melhor e com o passar do tempo haverá um equilíbrio ecológico muito presente nesse pequeno ecossistema.

Uma vez que a outra parte do parque ainda não recebeu nenhum tipo de infraestrutura o que pode ser comentadoé que passa por uma degradação ambiental muito forte, principalmente pela presença de lançamento de esgoto, in natura, diretamente nas águas do Córrego Barbado. Podemos visualizar no local a presença de espécies como: teiú, saguis, cágado d'água, cutia, capivara e várias espécies de pássaros.

$\mathrm{Na}$ medida em que os seres vivos que habitam o parque passam a ser reproduzir normalmente, o seu panorama visual mudará completamente e, com a adaptação desses seres vivos à presença constante do homem em sua volta, sem se preocupar em serem atacados por eles, essas espécies viverão em perfeita harmonia dentro desse local.

Outro ponto interessanteé a conservação da flora local, pois, sem o desmatamento e as queimadas habituais que aconteciam constantemente na região, haverá maiores condições das espécies que utilizam a flora como um todo se reproduzirem e habitaremna sem a necessidade da migração de uma região para outra em razão dos processos de degradação do meio ambiente local. Contudo, deve-se destacar também que as plantas do Cerrado, em determinado período, precisam da queimada para melhorar a sua constituição anatômica e fisiológica.

Tudo o que foi feito em relação à legalização da área para a criação de um parque estadual é válido, porém, devem ser realizadas outras ações dentro desse local ambiental para a conservação tanto da fauna quanto da flora, por meio de uma política ambiental voltada para a preservação do meio ambiente, para os frequentadores do parque e moradores da região.

Percebe-se na pesquisa em campo por meio de observações das espécies de aves, na pesquisa de dados em livros ou conversando com moradores dos bairros adjacentes ao parque estadual que a quantidade de aves presentes atualmente é menor que a de mais ou menos 20 anos. Esse fator se dá em razão da poluição, do desmatamento das matas ciliares próximas ao parque ou até mesmo em virtude das invasões que ocorreram nesse período, afetando por completo o ecossistema do local.

Muitos pássaros avistados no local apenas o utilizam como ponto de passagem ou dormitório, poucas espécies são vistas com frequência no parque, como é o caso do bem-te-vi, da rolinha, do pardal e do anu-preto. No entanto, uma pesquisa mais detalhada e crítica deve ser realizada no local, pois se acredita que mais espécies vivam no local.

Os animais de médio e grande porte são os menos avistados desde o desmatamento realizado na região da Grande Morada da Serra, primeiro para a construção do Centro Político Administrativo, que é a Sede do Governo Estadual, e suas Secretarias e depois para a construção dos Núcleos Habitacionais.

A quantidade de pássaros existente dentro do parque é outra incógnita, haja vista que, em muitos casos, como é de conhecimento de todos, temos espécies de hábito noturno e, por isso, deve-se realizar uma pesquisa mais profunda nesse ecossistema para verificar efetivamente as reais quantificações de espécies de seres vivos atuando no local.

Como alternativa para o aparecimento de aves pode ser criado um aviário nas dependências do parque estadual para estudo das espécies e cuidados relativos no que tange a doenças e assistência médica veterinária.

Outro ponto seria a criação de um local para o preparo de mudas, tanto para serem colocadas no parque quanto para distribuição aos visitantes, isso se daria em um espaço ao lado do parque e do miniestádio de futebol da Morada do Ouro.

Quanto à alimentação, o parque é um tanto carente, podendo ser vistas árvores frutíferas em pequenas quantidades e mesmo aquelas que existem não satisfazem todas as espécies, seja pelo tipo ou pela qualidade da fruta. São observadas as espécies: cumbaru, chico-magro, bocaiuva e outras espécies nativas, bem como bananeiras e alguns pés de mamão, mas em pequena quantidade. 
O que poderia ser feito de imediato é o plantio de algumas árvores frutíferas da região, tais como: mangueira, mamoeiro, acerola, laranjeiras e outras espécies que tenham um crescimento rápido para suprir a necessidade dos seres vivos do parque. Uma alternativa seria a colocação de alimentos em determinados pontos do parque, o que facilitaria a permanência desses seres dentro dele.

A criação de corredores ecológicos artificiais, ou seja, um corredor artificial transpondo as avenidas que passam entre o parque estadual pode seruma alternativa para a locomoção dos animais que fazem uso de árvores como ponto base de estadia ou mesmo de locomoção. Por exemplo, os saguis e os gambás são observados constantemente no crepúsculo da manhã ou da tarde fazendo tentativas de travessia. Em alguns casos foram encontrados saguis mortos nas proximidades.

Quanto às nascentes dentro do parque, deve-se fazer um estudo de impacto ambiental para definir o grau de comprometimento delas, pois, como se verifica a princípio, há erosão e assoreamento dessas nascentes.

\section{REFERÊNCIAS}

1. Câmara T, Murta R. Mamíferos da Serra do Cipó. Belo Horizonte: PUC-Minas, Museu de. Ciências Naturais; 2003.

2. Sabino J, Prado P. Perfil do Conhecimento da Diversidade de Vertebrados do Brasil. Brasília: Ministério do Meio Ambiente; 2000.

3. Silva CR. Riqueza e Diversidade de Mamíferos Não-Voadores em um Mosaico Formado por Plantios de Eucaliptus saligna e Remanescentes de Floresta Atlântica no Município de Pilar do Sul, São Paulo. [dissertação]. Piracicaba: Escola Superior de Agricultura Luiz Queiroz, Universidade São Paulo; 2001.

4. Zank S, Kindel A, Jardim M. Diferenciação de gatos domésticos e silvestres através de pegadas. $25^{\circ}$ Congresso Brasileiro de Zoologia; 2004; Brasília: Sociedade Brasileira de Zoologia; 2004. p. 229.

5. Oliveira TG, Cassaro K. Guia de Campo dos Felinos do Brasil. São Paulo: Instituto Pró-Carnívoros; Fundação Parque Zoológico de São Paulo; Sociedade de Zoológicos do Brasil; 2005.

6. Nascimento VL, Ferreira JÁ, Freitas DM, Souza
LL, Borges PA L, Tomas WM. Período de atividade de alguns vertebrados do pantanal, estimado por fotografia remota. $4^{\circ}$ Simpósio Sobre Recursos $\mathrm{Na}$ turais E Sócio-Econômicos Do Pantanal; Corumbá: EMBRAPA Pantanal; 2004. p. 1-4.

7. Primack RB, Rodrigues E. Biologia da conservação. Londrina: Midiograf; 2001.

8. Hickman CP, Roberts LS, Larson A. Princípios Integrados de Zoologia. 11a ed. Rio de Janeiro: Guanabara Koogan; 2004.

9. Sick H. Ornitologia brasileira. Rio de Janeiro: Nova Fronteira; 1997.

10. Brito C. Lei 7.313, 1 de setembro de 2000, criou a reserva ecológica do Centro Político Administrativo. Diário Oficial. 1 de setembro de 2000. [acesso 21 set. 2009]. Disponível em: http://www.al.mt.gov. br/V2008/ViewConteudo.asp?no_codigo=1155.

11. Brito C. Lei 7.506/01, de 21 de setembro de 2001. Será construído em área de 12 hectares, de um total de 59 hectares, abrigando trilhas para caminhadas, quadras poliesportivas, porto de ginástica e playground. Diário Oficial. 1 de setembro de 2000. [acesso 21 set. 2009]. Disponível em: http://www. al.mt.gov.br/V2008/ViewConteudo.asp?no_codigo $=1155$.

12. Abreu, EF. Parque Massairo Okamura. Diário de Cuiabá. 2004;(11007) [acesso 31 ago 2004]. Disponível em: http://www.diariodecuiaba.com.br/detalhe. php?cod $=192106$.

13. Prefeitura Municipal de Cuiabá. Conheça Cuiabá [Internet]. Cuiabá: Prefeitura Municipal; 2007. [acesso 31 ago 2007]. Disponível em: http://www.cuiaba. mt.gov.br/mapa.jsp.

14. Stape JL. Tectona grandis (teca) [nternet]. Piracicaba: IPEF; [acesso 21 jul. 2003]. Disponível em: http:/ / www.ipef.br/identificacao/tectona.grandis.asp.

Recebido: 27/12/2007

Received: $12 / 27 / 2007$

Aprovado: 18/02/2008

Approved: 02/18/2008 